\title{
Renin angiotensin system inhibitors for patients with stable coronary artery disease without heart failure: systematic review and meta-analysis of randomized trials
}

\author{
Sripal Bangalore, ${ }^{1}$ Robert Fakheri, ${ }^{1}$ Simon Wandel, ${ }^{3}$ Bora Toklu, ${ }^{2}$ Jasmin Wandel, ${ }^{4}$ Franz H Messerli5,6,7
}

${ }^{1}$ New York University School of Medicine, New York, NY, USA

${ }^{2}$ Mount Sinai Beth Israel

Medical Center, New York, NY, USA

${ }^{3}$ Novartis Pharma AG, Basel, Switzerland

4 Institute for Risks and Extremes, Bern University of Applied Sciences, Switzerland

5 University Hospital, Bern,

Switzerland

${ }^{6}$ Mount Sinai, Icahn School of Medicine, New York, NY, USA

${ }^{7}$ Jagiellonian University Krakow, Poland

Correspondence to: S Bangalore sripalbangalore@gmail.com

Cite this as: $B M J$ J 2017;356:j4 http://dx.doi.org/10.1136/bmj.j4

Accepted: 15 December 2016

\section{ABSTRACT}

OBJECTIVE

To critically evaluate the efficacy of renin angiotensin system inhibitors (RASi) in patients with coronary artery disease without heart failure, compared with active controls or placebo.

DESIGN

Meta-analysis of randomized trials.

\section{DATA SOURCES}

PubMed, EMBASE, and CENTRAL databases until 1 May 2016.

\section{ELIGIBILITY CRITERIA FOR SELECTING STUDIES}

Randomized trials of RASi versus placebo or active controls in patients with stable coronary artery disease without heart failure (defined as left ventricular ejection fraction $\geq 40 \%$ or without clinical heart failure). Each trial had to enroll at least 100 patients with coronary artery disease without heart failure, with at least one year's follow-up. Studies were excluded if they were redacted or compared use of angiotensin converting enzyme inhibitors with angiotensin receptor blockers. Outcomes were death, cardiovascular death, myocardial infarction, angina, stroke, heart failure, revascularization, incident diabetes, and drug withdrawal due to adverse effects. RESULTS

24 trials with 198275 patient years of follow-up were included. RASi reduced the risk of all cause mortality (rate ratio $0.84,95 \%$ confidence interval 0.72 to 0.98 ), cardiovascular mortality $(0.74,0.59$ to 0.94$)$, myocardial infarction $(0.82,0.76$ to 0.88$)$, stroke $(0.79,0.70$ to 0.89$)$, angina, heart failure, and revascularization when compared with placebo but not

\section{WHAT IS ALREADY KNOWN ON THIS TOPIC}

Renin angiotensin system inhibitors (RASi) are strongly recommended by guidelines (class I or Ila) for patients with coronary artery disease without heart failure, on the basis of early studies of RASi versus placebo showing significant reduction in cardiovascular events including mortality

However, later studies with improvement in background treatment showed no benefit of RASi versus placebo

\section{WHAT THIS STUDY ADDS}

In patients with stable coronary artery disease without heart failure, a significant benefit of RASi was seen for the reduction of cardiovascular events and all cause mortality only in comparison with placebo but not with active controls

Even among the placebo controlled trials, the benefits were only seen in trials with high baseline risk and not in those with low baseline risk

The blanket recommendation to use RASi for all patients with coronary artery disease is not supported by evidence

when compared with active controls (all cause mortality, 1.05, 0.94 to 1.17; $P_{\text {interaction }}=0.006$; cardiovascular mortality, 1.08, 0.93 to 1.25 , $P_{\text {interaction }}<0.001$; myocardial infarction, $0.99,0.87$ to 1.12, $P_{\text {interaction }}=0.01$; stroke, $1.10,0.93$ to 1.31 ; $\mathrm{P}_{\text {interaction }}=0.002$ ). Bayesian meta-regression analysis showed that the effect of RASi when compared with placebo on all cause mortality and cardiovascular mortality was dependent on the control event rate, such that RASi was only beneficial in trials with high control event rates $(>14.10$ deaths and $>7.65$ cardiovascular deaths per 1000 patient years) but not in those with low control event rates.

\section{CONCLUSIONS}

In patients with stable coronary artery disease without heart failure, RASi reduced cardiovascular events and death only when compared with placebo but not when compared with active controls. Even among placebo controlled trials in this study, the benefit of RASi was mainly seen in trials with higher control event rates but not in those with lower control event rates. Evidence does not support a preferred status of RASi over other active controls.

\section{Introduction}

Renin angiotensin system inhibitors (RASi) have been documented to reduce the risk of cardiovascular events and overall mortality when compared with placebo in patients with coronary artery disease and even in those without apparent heart failure. ${ }^{12}$ Because the mean systolic blood pressure on entry in these trials was lower than $140 \mathrm{~mm} \mathrm{Hg}$ and the end of trial difference in blood pressure between the two treatment strategy was minimal, the favorable effect of RASi on outcomes has been dubbed as a "blood pressure independent effect"-a vasculoprotective properties of these drugs. ${ }^{3}$

However, in the Prevention of Events with Angiotensin Converting Enzyme Inhibition (PEACE) trial of patients with stable coronary artery disease and normal or slightly reduced left ventricular function, RASi provided no further benefit when compared with placebo. ${ }^{4}$ Similar results with no benefit of RASi were seen in the Quinapril Ischemic Event Trial (QUIET) ${ }^{5}$, Comparison of Amlodipine vs Enalapril to Limit Occurrences of Thrombosis (CAMELOT) study, ${ }^{6}$ and Ischemia Management With Accupril Post-Bypass Graft via Inhibition of the Converting Enzyme (IMAGINE) trial. ${ }^{7}$ These seemingly incongruous findings were attributed to lower rate of events in these four trials than in the HOPE and EUROPA trials, ${ }^{12}$ owing to increased use of intense treatment including revascularization and lipid lowering treatment. 
Despite the above, the American College of Cardiology Foundation (ACCF)/American Heart Association (AHA) guidelines on stable ischemic heart disease recommends RASi in patients who also have hypertension, diabetes, left ventricular ejection fraction (LVEF) of $40 \%$ or less, or chronic kidney disease, unless contraindicated (class I, level A) or in patients with other vascular disease (class IIa). ${ }^{8}$ The objective of the current study was to critically evaluate the efficacy of RASi in patients with coronary artery disease without heart failure.

\section{Methods}

Database search and eligibility criteria

We searched PubMed, Cochrane Central Register of Controlled Trials (CENTRAL), and EMBASE until 1 May 2016, for randomized controlled trials of RASi (angiotensin converting enzyme inhibitors or angiotensin receptor blockers) in patients with coronary artery disease without heart failure. The MeSH terms used are outlined in table S1. There was no language restriction for the search. In addition, we searched the bibliographies of original trials, meta-analyses, and review articles identified to find other eligible trials, and kept up to date with the search by weekly reminders from PubMed.

Eligible trials had to fulfill the following criteria: compared RASi with placebo or active controls; enrolled at least 100 patients with coronary artery disease without heart failure (defined as LVEF $\geq 40 \%$ or without clinical heart failure) with follow-up of at least one year (to minimize small study effect); and reported the outcomes of interest (see below). We excluded studies if they were redacted for any reason or compared use of angiotensin converting enzyme inhibitors with angiotensin receptor blockers. Given that there was no patient recruitment, ethical approval was not required.

\section{Data extraction and bias assessment}

Three authors (RF, BT, SB) independently assessed trial eligibility and trial bias risk and extracted data. Disagreements were resolved by consensus. The trials' bias risk was assessed with the components recommended by the Cochrane Collaboration for randomized trials. ${ }^{9}$ These components include allocation sequence generation, allocation concealment, blinding of outcome assessors, incomplete outcome data, and selective outcome reporting. For each component, trials were categorized as low, high, or unclear risk of bias. The trials did not differ by the last two components, and thus we considered trials with high or unclear risk for bias for the first three components to represent trials with high risk of bias.

\section{Outcomes}

Primary outcomes evaluated were all cause mortality, cardiovascular death, myocardial infarction, stroke, angina pectoris, and heart failure. Secondary outcomes evaluated were revascularization, incident diabetes, and drug withdrawal due to adverse effects.

\section{Statistical analyses}

Trial level meta-analysis was performed as per the recommendations from the Cochrane Collaboration and the preferred reporting items for systematic reviews and meta-analyses (PRISMA) statement. ${ }^{910}$ We performed the analysis using an intention to treat approach. Analyses compared RASi versus placebo and RASi versus active controls separately. A test for interaction was used to compare the quantitative (magnitude) and qualitative direction of the effect size for the placebo and active control trials, with $\mathrm{P}_{\text {interaction }}<0.05$ considered statistically significant.

We calculated the meta-analytic summary estimates (rate ratio) using the fixed effect model and the random effects model of DerSimonian and Laird. ${ }^{11}$ Continuity correction was used for trials with zero events to enable finite variance estimators to be derived. ${ }^{12}$ Heterogeneity (proportion of total variation observed between the trials attributable to differences between trials rather than chance) was assessed using the $\mathrm{I}^{2}$ statistic $^{13}(<25 \%=$ low; $>75 \%=$ high). We assessed small study effect using the Begg's and the Egger's test and by visual evaluation of the funnel plots for asymmetry.

\section{Heterogeneity of treatment effect}

For the heterogeneity of treatment effect based on baseline risk, we calculated the event rate per 1000 patient years of follow-up in the control arm (placebo or active) of the trial. The control event rate is a good measure of not only the baseline clinical characteristic of the enrolled cohort (such as the proportion with diabetes or kidney disease), but also the background treatment (such as statin use). Conventional meta-regression of the treatment effect with the control event rate as explanatory variable is problematic because it ignores the correlation between the treatment effect and the control event rate. Therefore, a Bayesian meta-regression was used, which accounts appropriately for this correlation. ${ }^{1415}$

A strong interaction effect between the treatment effect and the baseline risk is indicated if the $95 \%$ credible interval for the control event rate parameter excludes zero. To further quantify this interaction, a credible band for the relation between the treatment effect and baseline risk was calculated, and the turning point for which there is no treatment effect (that is, rate ratio=1) was calculated. Vague priors were used for all parameters; we conducted all analyses using WinBUGS 1.4.3 and $\mathrm{R}$ 3.3.0.

We did a conventional meta-regression analysis to evaluate the relation of the following potential effect modifiers to the risk of outcomes with RASi: end of trial systolic blood pressure difference between treatment arms, percentage of patients with hypertension, percentage of patients with diabetes, and percentage of patients on statins. This was done separately for placebo controlled trials versus active controlled trials. We used a residual maximum likelihood to estimate the additive (between study) component of variance $\tau^{2}$ for the meta-regression analysis. Bootstrap analyses were performed with a Monte Carlo permutation test for meta-regression using 10000 random permutations. ${ }^{16}$ We used standard statistical software (Stata 12.1, Stata) to conduct analyses. ${ }^{17}$ 


\section{Sensitivity analysis}

Sensitivity analyses were performed for the following subgroups: trials with mean baseline systolic blood pressure $140 \mathrm{~mm} \mathrm{Hg}$ or less versus more than $140 \mathrm{~mm}$ $\mathrm{Hg}$ (to evaluate whether the benefit of RASi is restricted to patients with higher entry blood pressure); trials enrolling patients with recent myocardial infarction (defined as $\leq 3$ months) versus those not (to evaluate whether the benefit of RASi is restricted to patients with recent myocardial infarction); and trials using angiotensin converting enzyme inhibitors compared with those using angiotensin receptor blockers as the treatment.

\section{Trial sequential analysis}

To compare RASi with active controls, the lack of significant benefit of RASi could merely be due to type II error. To evaluate this, we used trial sequential analysis to evaluate whether the RASi versus active controls comparisons had adequate power. For trial sequential analysis, monitoring boundaries are drawn for each outcome, similar to interim analysis of randomized trials. This provides information on whether to continue evaluating for evidence when the boundary is not crossed or whether sufficient evidence is reached for anticipated effect or for futility when the boundary is crossed. Trial sequential analysis was performed by estimation of the required diversity adjusted information size (sample size), ${ }^{18}$ anticipating a $15-20 \%$ relative risk reduction for the outcome (death, cardiovascular death, stroke), $\alpha=5 \%$ and $1-\beta=90 \%$. The methodology has been described previously. ${ }^{1920}$ The $15-20 \%$ relative reduction was chosen because it is the nominal effect size seen in cardiovascular trials that is both clinically meaningful and realistic.

\section{Patient involvement}

No patients were involved in setting the research question or the outcome measures, nor were they involved in developing plans for design or implementation of the study. No patients were asked to advise on interpretation or writing up of results. There are no plans to disseminate the results of the research to study participants or the relevant patient community.

\section{Results}

\section{Study selection}

Our search included 24 trials that satisfied the inclusion and exclusion criteria (fig S1). The trials enrolled 61961 patients who were followed up for an average of 3.2 years, for a total of 198275 patient years of follow-up (tables S2-S3). Of the included trials, five enrolled patients within three months of acute myocardial infarction, 18 were placebo controlled, and seven were active controlled. The active comparison groups were calcium antagonists (four trials), thiazide diuretic (one), and conventional treatment (two). Nineteen trials had mean systolic blood pressure lower than $140 \mathrm{~mm} \mathrm{Hg}$ at baseline. The baseline characteristics and bias risk assessment of the trials are outlined in tables S2-S3.

\section{All cause mortality}

RASi reduced the risk of all cause mortality when compared with placebo (rate ratio $0.84,95 \%$ confidence interval 0.72 to 0.98 ) but not when compared with active controls (1.05, 0.94 to 1.17; $\mathrm{P}_{\text {interaction }}=0.006$; fig 1$)$. Bayesian meta-regression showed that the beneficial effect of RASi versus placebo on all cause mortality depended on the control event rate, such that RASi was only beneficial in trials with a high control event rate $(>14.10$ deaths per 1000 patient years) but not in those with a low control event rate (fig 2). The other effect modifiers were not significant predictors (tables S8-S9). Trial sequential analysis for RASi versus active controls showed that the cumulative $\mathrm{Z}$ curve did not cross either the conventional boundary or the trial sequential monitoring boundary, indicating lack of even a 15\% benefit of RASi over active controls with 90\% power (fig S3). There was moderate statistical heterogeneity in the comparison versus placebo but no statistical heterogeneity in the comparison with active controls (fig 1).

\section{Cardiovascular mortality}

RASi reduced the risk of cardiovascular mortality when compared with placebo (rate ratio $0.74,95 \%$ confidence interval 0.59 to 0.94 ) but not when compared with active controls (1.08, 0.93 to 1.25; $\mathrm{P}_{\text {interaction }}<0.001$; fig 3). Bayesian meta-regression showed that the beneficial effect of RASi versus placebo on cardiovascular mortality depended on the control event rate such that RASi was only beneficial in trials with a high control event rate ( $>7.65$ cardiovascular deaths per 1000 patient years) but not in those with a low control event rate (fig 4). The other effect modifiers were not significant predictors (tables S8-S9). Trial sequential analysis for RASi versus active controls showed that the cumulative $Z$ curve did not cross either the conventional boundary or the trial sequential monitoring boundary, indicating lack of even a 15\% benefit of RASi over active controls with $90 \%$ power (fig S4). There was moderate statistical heterogeneity in the comparison of RASi versus placebo, but no statistical heterogeneity in the comparison with active controls (fig 4).

\section{Myocardial infarction}

RASi reduced the risk of myocardial infarction when compared with placebo (rate ratio $0.82,95 \%$ confidence interval 0.76 to 0.88 ) but not when compared with active controls $\left(0.99,0.87\right.$ to 1.12 ; $P_{\text {interaction }}=0.01$; fig 5$)$. Bayesian meta-regression showed that the beneficial effect of RASi versus placebo on myocardial infarction depended on the control event rate such that RASi was beneficial in trials with a high control event rate but not in those with a low control event rate (fig 6). However, the 95\% credibility for $\beta$ included zero ( -0.38 to 0.15$)$. Moreover, the end of trial systolic blood pressure difference between RASi and placebo explained $93.1 \%$ of the between study variance-that is, RASi was beneficial when compared with placebo mainly in trials with a greater difference in systolic blood pressure at the end of the trial (fig S3). Trial sequential analysis for RASi versus active controls showed that the cumulative $\mathrm{Z}$ curve did not cross either the conventional boundary or 
Fig 1 | Forest plot showing effect of renin angiotensin system inhibitors (RASi) versus placebo or active controls on all cause mortality in patients with stable coronary artery disease without heart failure. $\mathrm{D}+\mathrm{L}=$ DerSimonian and Laird; I-V=inverse variance; $\mathrm{CHD}=$ coronary heart disease
Trials

Placebo

APRES

CAMELOT

CATS

Cai et al

EUROPA

HOPE

IMAGINE

JAMP

Kondo et al

PART-2

PEACE

PRACTICAL

PREAMI

QUIET

QUO VADIS

ROADMAP (CHD subgroup)

SCAT

TRANSCEND

$D+L$ subtotal: $P=0, I^{2}=66.8 \%$

I-V subtotal

Active

ALLHAT (CHD subgroup)

CAMELOT

CARP

HIJ-CREATE

JMIC-B

NAGOYA HEART (CHD subgroup)

OLIVUS

$D+L$ subtotal: $P=0.983, I^{2}=0 \%$

I-V subtotal

$D+L$ overall: $P=0, I^{2}=59.9 \%$

I-V overall

$P_{\text {interaction }}$ (placebo $v$ active $)=0.006$
No of events/total

RASi Control

\section{$2 / 80$}

$8 / 673$

$2 / 149$

$47 / 478$

$375 / 6110$

$482 / 4645$

$28 / 1280$

$47 / 422$

$4 / 203$

$16 / 308$

$299 / 4158$

$12 / 150$

$40 / 631$

$27 / 878$

$0 / 75$

$13 / 564$

$8 / 229$

$364 / 2954 \quad 349 / 2972$

10

$\begin{array}{cc}407 / 2270 & 1067 / 6145 \\ 8 / 673 & 7 / 663 \\ 7 / 90 & 6 / 101 \\ 69 / 1024 & 59 / 1025 \\ 15 / 822 & 12 / 828 \\ 6 / 158 & 8 / 174 \\ 4 / 126 & 4 / 121\end{array}$

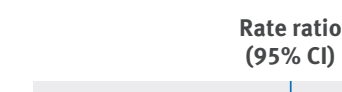

$$
\begin{aligned}
& 8 / 79 \\
& 6 / 655
\end{aligned}
$$

$$
3 / 149
$$

$99 / 344$

$420 / 6108$

$569 / 4652$

$28 / 1273$

$47 / 466$

$11 / 203$

$25 / 309$

$334 / 4132$

$12 / 75$

$37 / 621$

$27 / 872$

$0 / 73$

$3 / 540$

$11 / 231$

$349 / 2972$

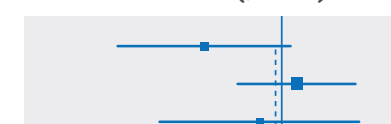

$t$

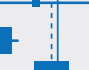

-
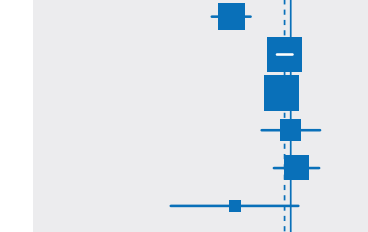

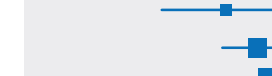

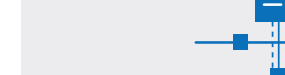

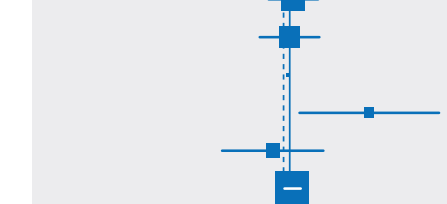

\section{.}
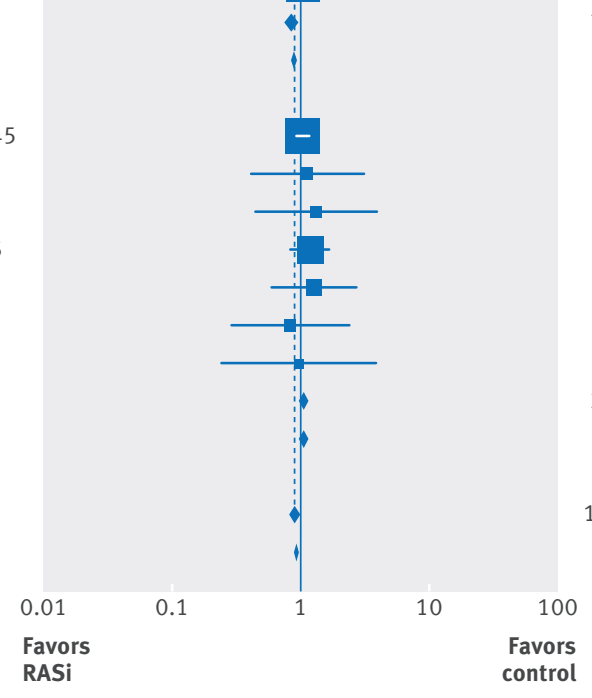

avors ntrol $\begin{array}{cc}\begin{array}{c}\text { Weight } \\ (\%)\end{array} & \text { Rate ratio } \\ (95 \% \mathrm{Cl})\end{array}$

0.610 .25 (0.05 to 1.16 )

1.231 .30 (0.45 to 3.74$)$

0.460 .67 (0.11 to 3.99)

6.190 .34 (0.24 to 0.48$)$

10.430 .89 (0.78 to 1.03$)$

10.780 .85 (0.75 to 0.96 )

3.821 .00 (0.59 to 1.68$)$

$5.28 \quad 1.10$ (0.74 to 1.65$)$

1.070 .36 (0.12 to 1.14$)$

2.950 .64 (0.34 to 1.20 )

10.090 .89 (0.76 to 1.04$)$

$2.00 \quad 0.50$ (0.23 to 1.11$)$

4.691 .06 (0.68 to 1.66 )

3.730 .99 (0.58 to 1.69 )

$0.10 \quad 0.97$ (0.02 to 49.05)

$0.90 \quad 4.15$ (1.18 to 14.56$)$

1.610 .73 (0.30 to 1.82 )

10.281 .05 (0.91 to 1.22$)$

76.240 .84 (0.72 to 0.98)

0.88 (0.83 to 0.94$)$

10.91 .03 (0.92 to 1.16$)$

1.331 .13 (0.41 to 3.11)

$1.17 \quad 1.31$ (0.44 to 3.90 )

6.181 .17 (0.83 to 1.66 )

$2.19 \quad 1.26$ (0.59 to 2.69)

1.230 .83 (0.29 to 2.38 )

0.750 .96 (0.24 to 3.84)

23.761 .05 (0.94 to 1.17 )

1.05 (0.94 to 1.17$)$

$100.00 \quad 0.89$ (0.79 to 1.01$)$ 0.92 (0.88 to 0.98 ) the trial sequential monitoring boundary, indicating lack of even a $20 \%$ benefit of RASi over active controls with $90 \%$ power (fig S5). There was no statistical heterogeneity in the analysis (fig 5).

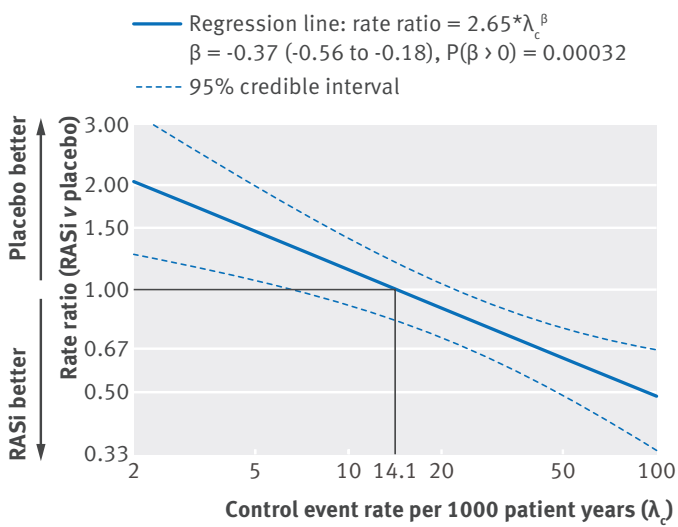

Fig 2 | Bayesian meta-regression analysis of the influence of baseline risk (control event rate) on the effect size of RASi versus placebo on all cause mortality
Stroke

RASi reduced the risk of stroke when compared with placebo (rate ratio $0.79,95 \%$ confidence interval 0.70 to 0.89 ) but not when compared with active controls (1.10, 0.93 to 1.31 ; $\mathrm{P}_{\text {interaction }}=0.002$; fig 7 ). Bayesian meta-regression showed that the beneficial effect of RASi versus placebo on stroke depended on the control event rate such that RASi was beneficial in trials with a high control event rate but not in those with a low control event rate. However, the $95 \%$ credibility for $\beta$ included zero ( -0.69 to 0.06$)$. The other effect modifiers were not significant predictors (tables S8-S9). There was no or low statistical heterogeneity in the analysis (fig 7).

\section{Angina}

RASi reduced the risk of angina when compared with placebo (rate ratio $0.94,95 \%$ confidence interval 0.89 to 0.99 ) but not when compared with active controls (1.07, 0.85 to 1.35 ; $\mathrm{P}_{\text {interaction }}=0.03$; fig 8 ). The other effect modifiers were not significant predictors (tables S8-S9). There was low statistical heterogeneity in the 
Fig 3 | Forest plot showing effect of renin angiotensin system inhibitors (RASi) versus placebo or active controls on cardiovascular mortality in patients with stable coronary artery disease without heart failure. $\mathrm{D}+\mathrm{L}=$ DerSimonian and Laird; I-V=inverse variance; $\mathrm{CHD}=$ coronary heart disease

\section{Trials}

Placebo

APRES

CAMELOT

Cai et al

EUROPA

HOPE

IMAGINE

JAMP

Kondo et al

PART-2

PEACE

PRACTICAL

QUIET

QUO VADIS

ROADMAP (CHD subgroup)

SCAT

TRANSCEND

$D+L$ subtotal: $P=0, I^{2}=73.2 \%$

I-V subtotal

Active

ALLHAT (CHD subgroup)
CAMELOT
CARP
HIJ-CREATE
JMIC-B
NAGOYA HEART (CHD subgroup)
OLIVUS
D+L subtotal: $P=0.853, \mathrm{I}^{2}=0 \%$
I-V subtotal
D+L overall: $P=0, I^{2}=68.2 \%$
I-V overall
$P_{\text {interaction }}$ (placebo $v$ active) $=0.0008$

No of events/total

RASi Control

$\begin{array}{cc}1 / 80 & 7 / 79 \\ 5 / 673 & 2 / 655 \\ 43 / 478 & 99 / 344 \\ 215 / 6110 & 249 / 6108 \\ 282 / 4645 & 377 / 4652 \\ 18 / 1280 & 15 / 1273 \\ 11 / 422 & 14 / 466 \\ 2 / 203 & 9 / 203 \\ 8 / 308 & 18 / 309 \\ 146 / 4158 & 152 / 4132 \\ 9 / 150 & 12 / 75 \\ 12 / 878 & 27 / 872 \\ 0 / 75 & 0 / 73 \\ 11 / 564 & 1 / 540 \\ 4 / 229 & 7 / 231 \\ 227 / 2954 & 223 / 2972\end{array}$

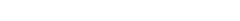

(215/2070

$215 / 2270 \quad 534 / 6145$

$5 / 673 \quad 5 / 663$

$0 / 90 \quad 0 / 101$

$28 / 1024 \quad 25 / 1025$

$6 / 822 \quad 6 / 828$

$0 / 158 \quad 4 / 174$

$1 / 126 \quad 2 / 121$

$4 / 174$
$2 / 121$

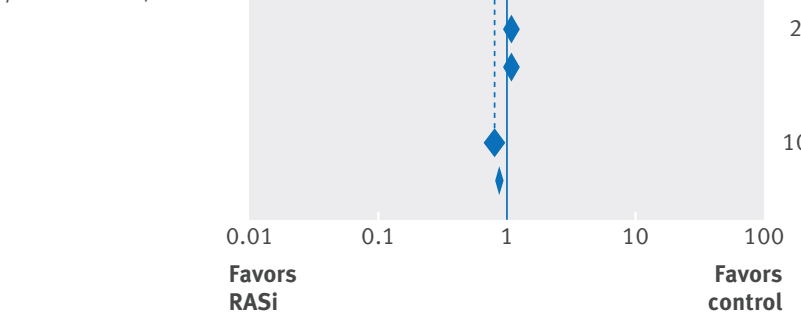

Rate ratio
$(95 \% \mathrm{Cl})$

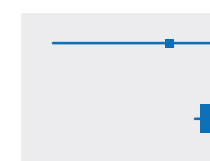

$\begin{array}{cc}\begin{array}{c}\text { Weight } \\ (\%)\end{array} & \text { Rate ratio } \\ (95 \% \mathrm{Cl})\end{array}$

$0.78 \quad 0.14$ (0.02 to 1.15$)$

1.222 .43 (0.47 to 12.54$)$

$8.40 \quad 0.32$ (0.22 to 0.45$)$

10.750 .86 (0.72 to 1.04$)$

11.050 .75 (0.64 to 0.87 )

4.721 .19 (0.60 to 2.37)

3.940 .87 (0.39 to 1.91$)$

$1.38 \quad 0.22$ (0.05 to 1.03 )

3.660 .45 (0.19 to 1.03 )

10.20 .96 (0.76 to 1.20$)$

3.470 .38 (0.16 to 0.89 )

3.970 .92 (0.42 to 2.01)

$0.23 \quad 0.97$ (0.02 to 49.05)

0.8110 .53 (1.36 to 81.58 )

2.020 .58 (0.17 to 1.97 )

10.721 .02 (0.85 to 1.23 )

77.320 .74 (0.59 to 0.94$)$

0.81 (0.75 to 0.89 )

11.011 .09 (0.93 to 1.28$)$

1.990 .99 (0.29 to 3.40)

0.231 .12 (0.02 to 56.56 )

$6.12 \quad 1.12$ (0.65 to 1.92 )

$2.31 \quad 1.01$ (0.33 to 3.12)

0.410 .12 (0.01 to 2.27 )

$0.60 \quad 0.48$ (0.04 to 5.30$)$

22.681 .08 (0.93 to 1.25$)$

1.08 (0.93 to 1.25$)$

$100.00 \quad 0.80$ (0.66 to 0.97$)$

0.87 (0.81 to 0.94$)$

Favors
RASi

control comparison versus placebo but moderate statistical heterogeneity in the comparison with active controls (fig 8).

\section{Heart failure}

RASi reduced the risk of heart failure when compared with placebo (rate ratio $0.78,95 \%$ confidence interval 0.71 to 0.86 ) but not when compared with active controls (0.89, 0.62 to 1.29; $P_{\text {interaction }}=0.49$; fig 9). The other effect modifiers were not significant predictors (tables S8-S9). There was moderate statistical heterogeneity in the comparison versus placebo but no statistical heterogeneity in the comparison with active controls (fig 9).

Revascularization, incident diabetes, and drug withdrawal due to adverse effects

RASi reduced the risk of revascularization when compared with placebo (rate ratio 0.93, 95\% confidence interval 0.89 to 0.98 ) but not when compared with active controls (1.02, 0.94 to 1.12; $P_{\text {interaction }}=0.07$; fig S6). The other effect modifiers were not significant predictors (tables S8-S9). There was low statistical heterogeneity in the analysis (fig S6). RASi also reduced the risk of incident diabetes when compared with placebo (0.84, 0.76 to 0.92$)$ and with active controls $(0.39,0.16$ to 0.93; $\mathrm{P}_{\text {interaction }}=0.09$; fig S7). There was moderate statistical heterogeneity in the analysis (fig S7). When

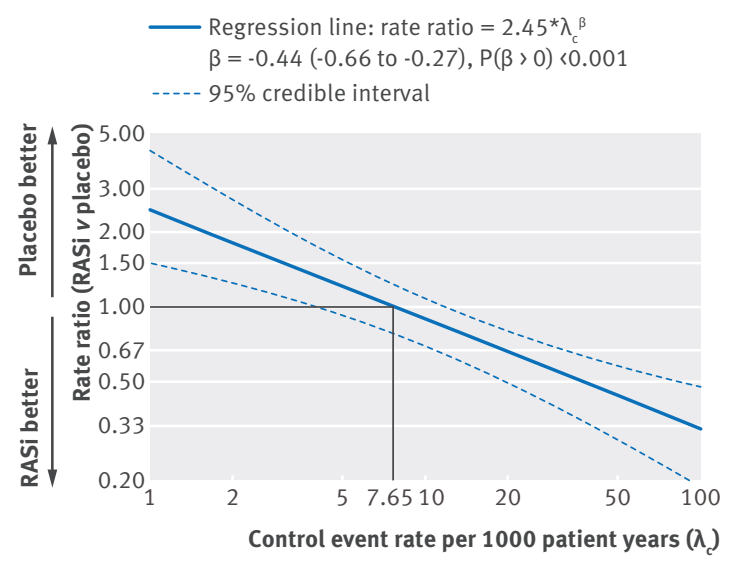

Fig 4 | Bayesian meta-regression analysis of the influence of baseline risk (control event rate) on the effect size of RASi versus placebo on cardiovascular mortality 


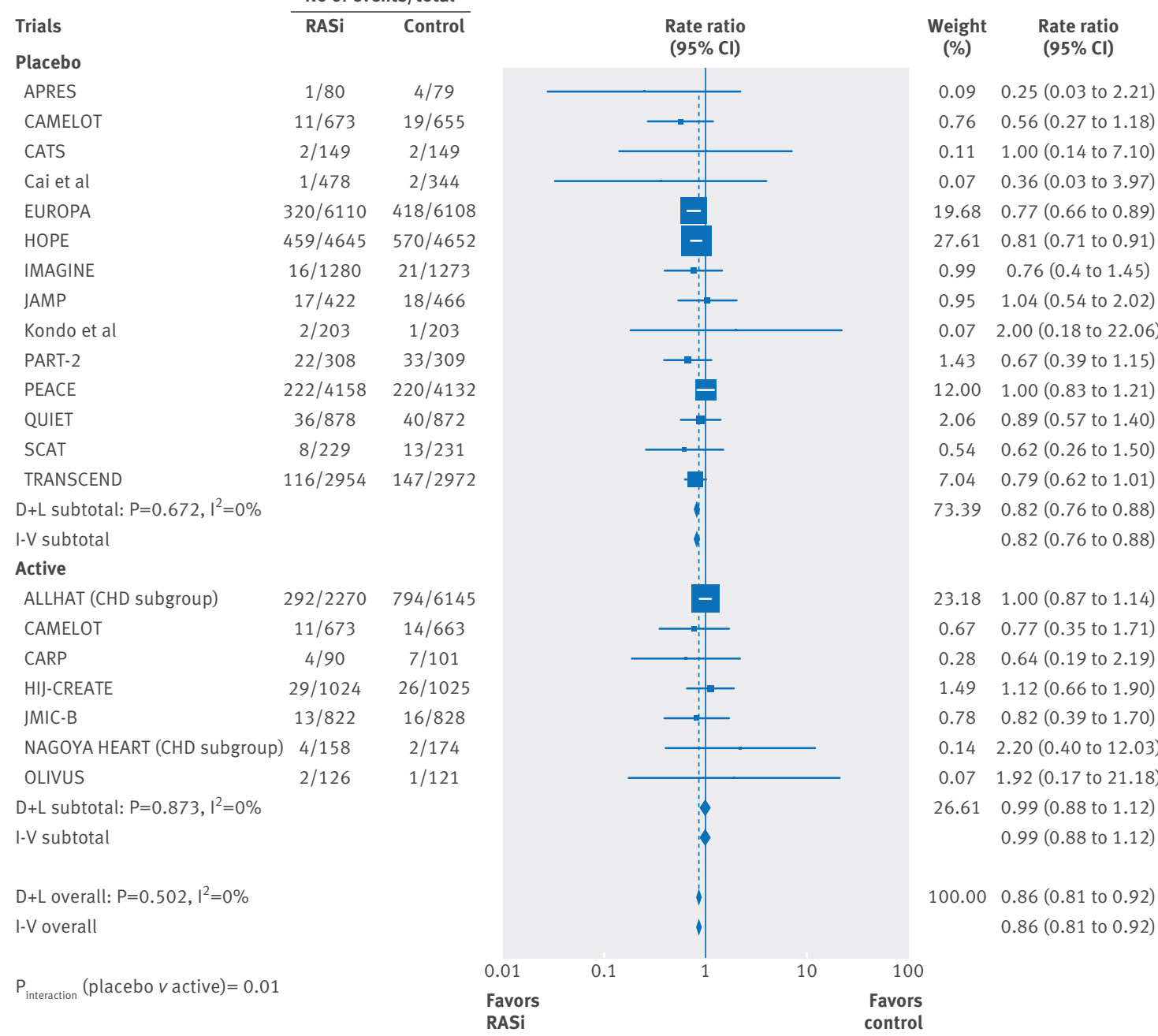

Fig 5 | Forest plot showing effect of renin angiotensin system inhibitors (RASi) versus placebo or active controls on myocardial infarction in patients with stable coronary artery disease without heart failure. $\mathrm{D}+\mathrm{L}=$ DerSimonian and Laird; $\mathrm{I}-\mathrm{V}=$ =inverse variance; $\mathrm{CHD}=$ coronary heart disease

compared with controls, there was a significant increase in the risk of drug withdrawal due to adverse effects with angiotensin converting enzyme inhibitors (1.57, 1.25 to 1.99 ) but not with angiotensin receptor blockers (0.66, 0.34 to 1.28; $\mathrm{P}_{\text {interaction }}<0.001$; fig S8; table S4). There was high statistical heterogeneity in the analysis (fig S8).

There was no significant small study effect in all the analyses.

\section{Sensitivity analysis}

For placebo controlled trials, the beneficial effect of RASi was independent of systolic blood pressure at baseline ( $\mathrm{P}_{\text {interaction }}>0.05$; table $\left.\mathrm{S} 5\right)$. The benefit of RASi over placebo was seen both in trials that enrolled patients with recent myocardial infarction as well as those that enrolled patients with stable ischemic heart disease $\left(P_{\text {interaction }}>0.05\right.$; table $\left.S 5\right)$. However, for the outcomes of death $\left(\mathrm{P}_{\text {interaction }}=0.004\right)$ and cardiovascular death $\left(P_{\text {interaction }}<0.001\right)$, the benefit was greater in patients with recent myocardial infarction than in those with stable ischemic heart disease (table S5).
For active control trials, there was a lack of benefit of RASi regardless of systolic blood pressure at baseline $\left(\mathrm{P}_{\text {interaction }}>0.05\right.$; table S6). Of note, none of the trials

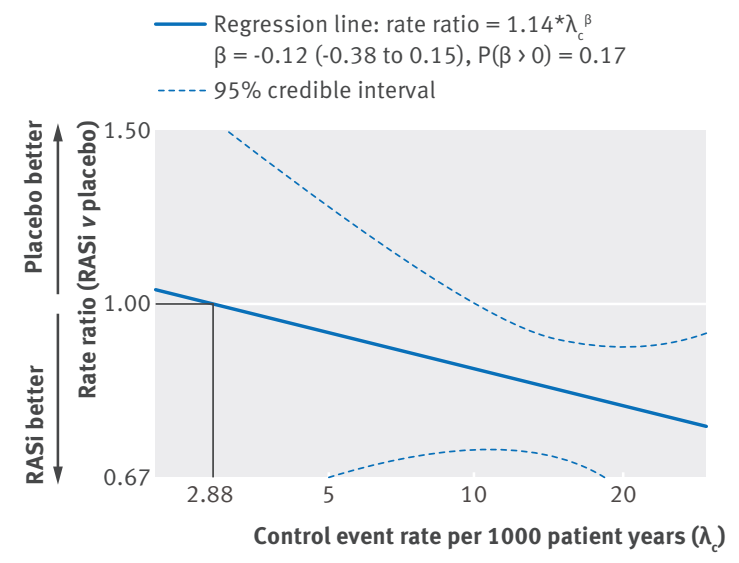

Fig 6 | Bayesian meta-regression analysis of the influence of baseline risk (control event rate) on the effect size of RASi versus placebo on myocardial infarction 


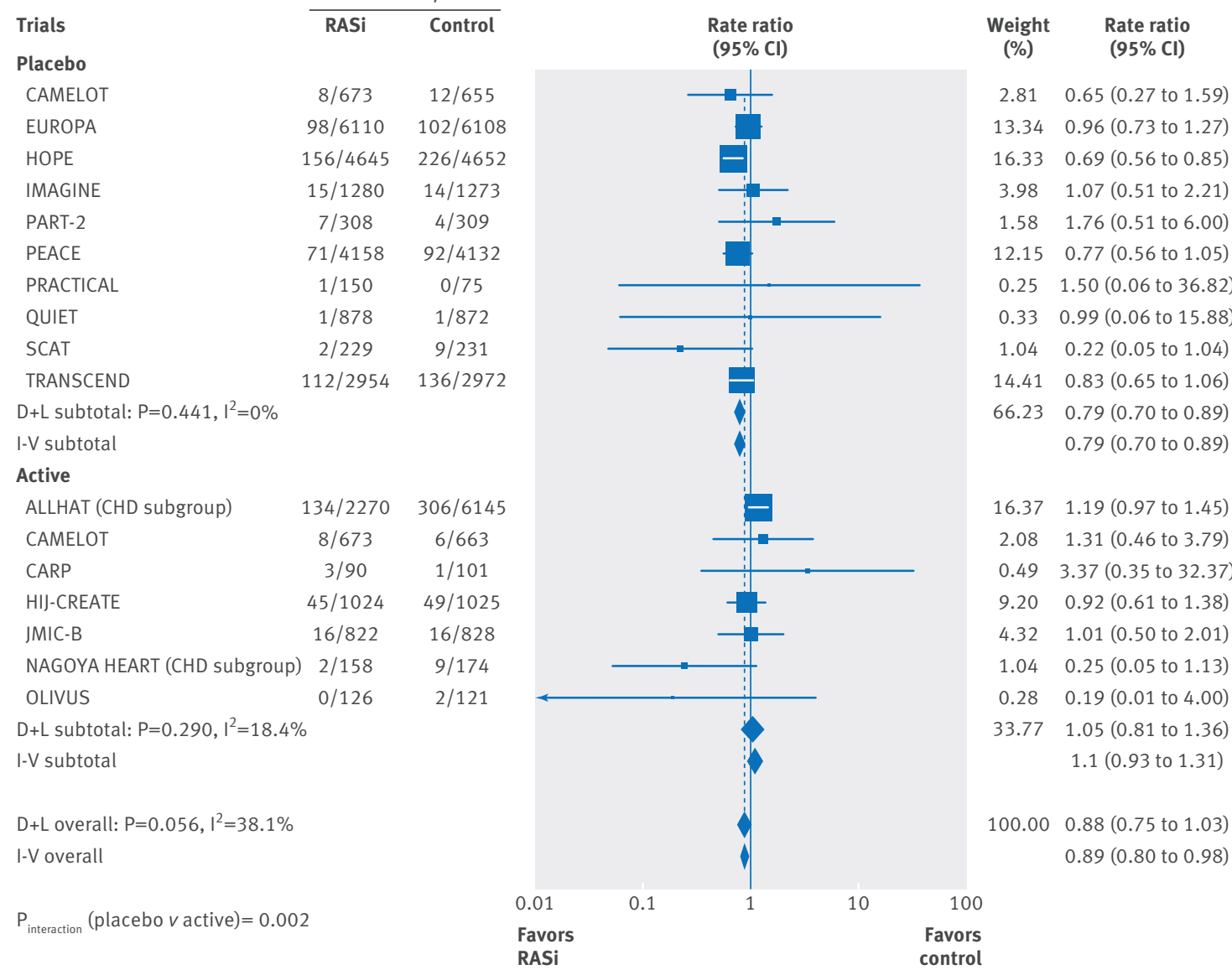

Fig 7 | Forest plot showing effect of of renin angiotensin system inhibitors (RASi) versus placebo or active controls on stroke in patients with stable coronary artery disease without heart failure. $\mathrm{CHD}=$ coronary heart disease

comparing RASi versus active controls enrolled patients with recent myocardial infarction. There was no statistically significant difference between angiotensin converting enzyme inhibitors versus active controls or angiotensin receptor blockers versus active controls for any of the efficacy outcomes (table S7). Angiotensin converting enzyme inhibitors were associated with an increase in angina when compared with active controls but only in the fixed effect model, not in the random effects model (table S7).

\section{Discussion}

This analysis of patients with stable coronary artery disease and without heart failure included data from 198275 patient years of follow-up from randomized trials. Although RASi reduced the risk of cardiovascular events (including all cause mortality) when compared with placebo, no such benefit was seen when compared with active controls. Moreover, even among the RASi versus placebo trials, the beneficial effect of RASi was restricted to trials with higher baseline risk but not in those with lower baseline risk.

RASi for patients with stable coronary artery disease without heart failure

In 2000, the landmark HOPE trial showed an impressive $22 \%$ reduction in the primary composite outcome, and reductions in death (16\%), cardiovascular death (26\%), myocardial infarction (20\%), stroke (32\%), and heart failure (23\%) with RASi (ramipril) when compared with placebo in 9297 high risk patients. ${ }^{1}$ Three years later, the EUROPA trial showed a similar benefit with RASi (perindopril), with a 20\% reduction in the primary composite endpoint and $22 \%$ reduction in myocardial infarction when compared with placebo in 12218 patients with stable coronary artery disease and no apparent heart failure. ${ }^{2}$

However, other trials subsequently failed to show a similar benefit. In the PEACE trial (2004) of 4158 patients with stable coronary artery disease and normal or slightly reduced left ventricular function, RASi (perindopril) was no better than placebo at reducing cardiovascular events. ${ }^{4}$ Similarly, in the QUIET trial (2001) of patients after coronary angioplasty but without systolic left ventricular dysfunction, no benefit of RASi (quinapril) was seen when compared with placebo. ${ }^{5}$ In CAMELOT, the calcium antagonist amlodipine reduced adverse cardiovascular events in patients with coronary artery disease and normal blood pressure, whereas RASi (enalapril), despite a similar blood pressure reduction, was no better than placebo. ${ }^{6}$ Finally, no benefit with RASi (quinapril) over placebo was seen in the IMAGINE trial (2008) of patients after bypass graft surgery. ${ }^{7}$ 


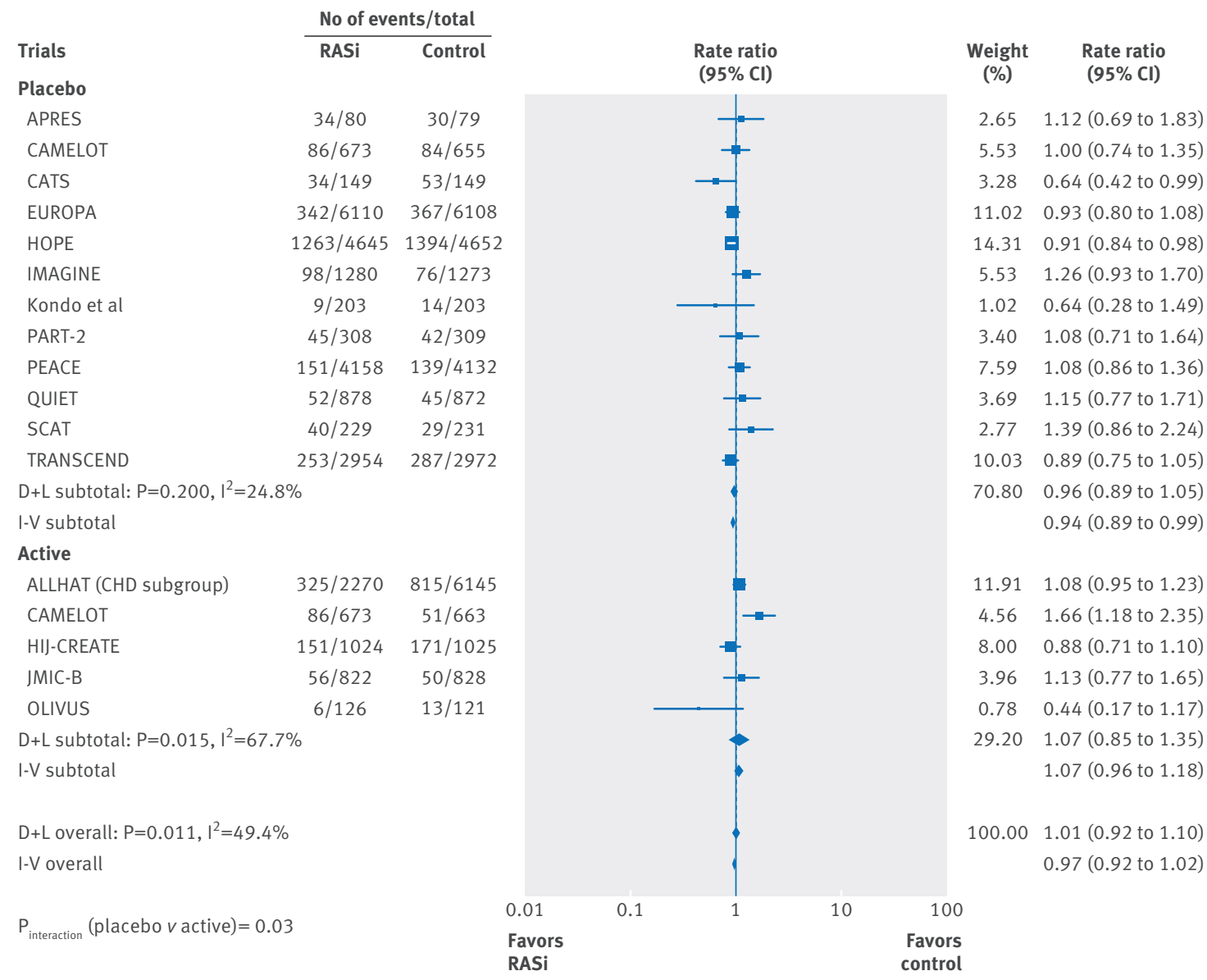

Fig 8 | Forest plot showing effect of renin angiotensin system inhibitors (RASi) versus placebo or active controls on angina pectoris in patients with stable coronary artery disease without heart failure. $\mathrm{CHD}=$ coronary heart disease

The lack of benefit in later trials could be due to lower event rate in these trials than those in the HOPE/ EUROPA trials, owing to increased use of revascularization and lipid lowering treatment. However, the 2012 ACCF/AHA guidelines continue to give a class I recommendations for RASi use in patients with stable coronary artery disease regardless of revascularization status or lipid lowering treatment therapy use in patients who also have hypertension, diabetes, LVEF of $40 \%$ or less, or chronic kidney disease and a class IIa recommendation for those with stable coronary artery disease and other vascular disease. We therefore explored the question as to the strength of the evidence to support RASi use in patients with coronary artery disease without heart failure. In addition, given the availability of other agents such as diuretics, $\beta$ blockers and calcium channel blockers, we specifically explored RASi use compared with active controls.

Our analysis with data from about 200000 patient years of follow-up offers some insights:

- RASi reduced the risk of cardiovascular events and all cause mortality when compared with placebo, similar to the results seen in HOPE and EUROPA. However, even in the placebo controlled trials, Bayesian meta-regression analysis showed that the baseline risk (as measured by control event rate) explained the heterogeneity of treatment effect such that RASi was only beneficial in trials with a higher baseline risk and not those with a lower baseline risk. Our results are consistent with the negative trials such as the QUIET, PEACE, CAMELOT, and IMAGINE trials. ${ }^{4-7}$ Thus the universal endorsement of RASi for all patients with stable coronary artery disease is not supported by even placebo controlled trials, and might apply only to patients with higher baseline risk.

- RASi did not reduce the risk of cardiovascular events or mortality when compared with active controls. With 14220 patients and 45504 patient years of follow-up, the trial sequential analysis showed that the lack of superiority of RASi over active controls was not due to trials being underpowered, because the analyses (for mortality, cardiovascular mortality, and myocardial infarction) had more than $90 \%$ power to show a $15-20 \%$ reduction in endpoints with RASi.

- Results were consistent between trials of angiotensin converting enzyme inhibitors and those of angiotensin receptor blockers when compared with active controls.

- There was no outcome for which a higher percentage of participants with either enrolled hypertension or diabetes conferred a statistically significant advantage of RASi over active controls.

- RASi showed a consistent reduction in incident diabetes when compared with placebo or active controls. 


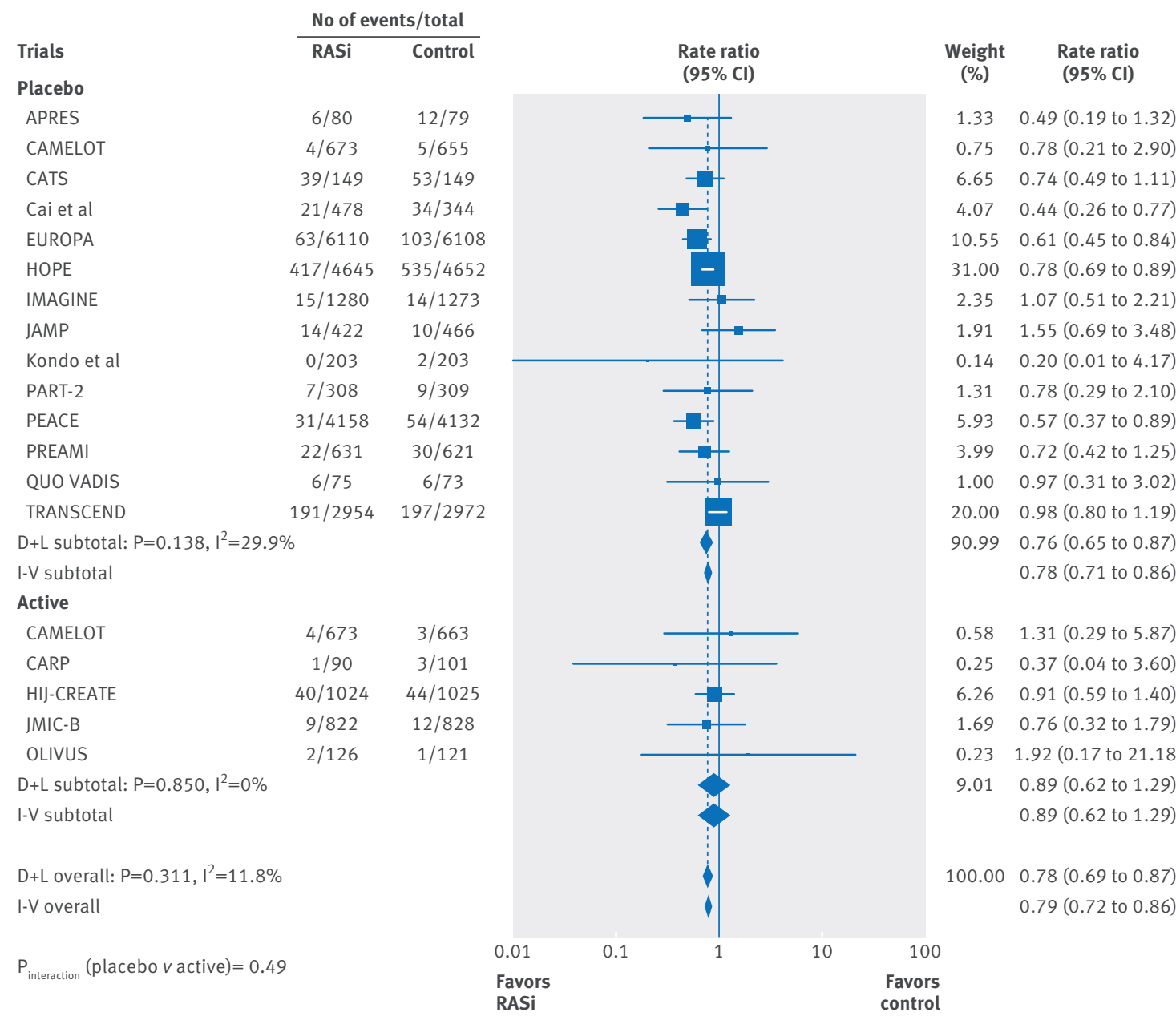

Fig 9 | Forest plot showing effect of renin angiotensin system inhibitors (RASi) versus placebo or active controls on heart failure in patients with stable coronary artery disease without heart failure on trial entry

Other meta-analysis in patients with hypertension have shown that RASi reduces the risk of new onset diabetes compared with other antihypertensive drugs. ${ }^{21}$

- Among RASi, angiotensin converting enzyme inhibitors had a higher withdrawal rate due to adverse events than controls, whereas angiotensin receptor blockers did not.

The lack of advantage of RASi over active controls for cardiovascular events could be due to three reasons. Firstly, the active controls are as good as RASi, and the effect is mediated mainly by a reduction in blood pressure. Secondly, the enrolled cohort (unlike patients with heart failure or renovascular hypertension) might not have had an activated renin-angiotensin-aldosterone system, resulting in less benefit. And finally, in this cohort, the renin-angiotensin-aldosterone system could have had a role in delaying the chronic process of atherogenesis and the benefit might not have been apparent during the short follow-up in these trials.

Therefore, our findings have important implications for clinical practice; they do not support the broad use of RASi for all patients with stable ischemic heart disease as has been recommended by current guidelines. The class I recommendation for patients with stable coronary artery disease and either diabetes or hypertension is not fully supported by the data in that the outcomes were similar to active comparators. Even among placebo controlled trials, RASi was no better than placebo in trials with lower baseline risk. This lack of benefit could have implications for patients with stable coronary artery disease with aggressive management of risk factors such as hypertension and hypercholesterolemia (with high intensity statins) and consequent lower baseline residual risk.

However, the current study did not evaluate other class I indications for RASi such as in patients with LVEF of $40 \%$ or less (where there is evidence of significant benefit of RASi) or in those with chronic kidney disease. Similarly, the class IIa recommendation for patients with stable coronary artery disease and other vascular diseases is also not fully supported by the data in that the outcomes were similar to active comparators. This begs the question as to the evidence to support the preferential recommendation for RASi for this indication. Similar results were seen in an analysis from the Reduction of Atherothrombosis for Continued Health (REACH) registry, where the use of angiotensin converting enzyme inhibitors or angiotensin receptor blockers was not associated with better outcomes in 
20909 outpatients with stable coronary artery disease and free of clinical heart failure at baseline. ${ }^{22}$

\section{Study limitations}

This meta-analysis had trial level data and hence we were unable to control for between trial differences. Not all trials had data on ejection fraction for all patients. Although many trials included patients based on an ejection fraction threshold, others based their inclusion on absence of clinical heart failure or an ejection fraction threshold. These results do not apply to cohorts not included in the present study, such as those with heart failure and chronic kidney disease. Not all of the trials reported each of the outcomes tested. Moreover, the active comparators were mainly calcium antagonists, and the results should not be extrapolated to other drugs not tested in the included trials.

\section{Conclusions}

In patients with stable coronary artery disease without heart failure, the current body of evidence from randomized trials shows a significant benefit of RASi for the reduction of cardiovascular events and all cause mortality only in comparison with placebo but not with active controls. Moreover, even among the placebo controlled trials, the benefit of RASi was only seen in trials with high baseline risk and not in those with low baseline risk. Therefore, the blanket recommendation to use RASi for all patients with coronary artery disease is not supported by evidence.

Contributors: SB was responsible for the study concept and design, and supervised the study. RF, BT, and SB were responsible for acquisition of data. SB, RF, BT, and FHM were responsible for analysis and interpretation of data. SB drafted the manuscript, and SB, RF, BT, and FHM undertook for critical revision of the manuscript for important intellectual content. SB, SW, and JW did the statistical analysis. SB is the study guarantor, had full access to all of the trial level data in the study, takes responsibility for the integrity of the data, and accuracy of the data analysis, and had the final responsibility to submit for publication. Funding: This work was not funded and hence there was no role of any funding source in the conception, data synthesis, analysis, interpretation, or in drafting of the manuscript.

Competing interests: All authors have completed the ICMJE uniform disclosure form at www.icmje.org/coi_disclosure.pdf and declare: no support from any organisation for the submitted work; SB declares honorariums from Daiichi-Sankyo, Pfizer, Abbott, Merck, BoerhingerIngelheim, Gilead, and Abbott-Vascular; SW is employed and hold shares at Novartis Pharma AG (Basel, Switzerland); FHM declares honorariums from Daiichi-Sankyo, Pfizer, Abbott, Servier, WebMD, Ipca, American College of Cardiology, Menarini, and Relypsa; the remaining authors have no relevant disclosures; none of the authors received any compensation for their work on this manuscript.

Ethical approval: Not required.

Data sharing: No additional data available.

The lead author affirms that the manuscript is an honest, accurate, and transparent account of the study being reported; that no important aspects of the study have been omitted; and that any discrepancies from the study as planned (and, if relevant, registered) have been explained.

This is an Open Access article distributed in accordance with the Creative Commons Attribution Non Commercial (CC BY-NC 3.0) license, which permits others to distribute, remix, adapt, build upon this work non-commercially, and license their derivative works on different terms, provided the original work is properly cited and the use is noncommercial. See: http://creativecommons.org/licenses/by-nc/3.0/.

1 Yusuf S, Sleight P, Pogue J, Bosch J, Davies R, Dagenais G. The Heart Outcomes Prevention Evaluation Study Investigators. Effects of an angiotensin-converting-enzyme inhibitor, ramipril, on cardiovascular events in high-risk patients. N Engl / Med 2000;342:145-53. doi:10.1056/NEJM200001203420301.
2 Fox KM. EURopean trial On reduction of cardiac events with Perindopril in stable coronary Artery disease Investigators. Efficacy of perindopril in reduction of cardiovascular events among patients with stable coronary artery disease: randomised, double-blind, placebo-controlled, multicentre trial (the EUROPA study). Lancet 2003;362:782-8. doi:10.1016/S0140-6736(03)14286-9.

3 Yusuf S, Lonn E.Anti-ischaemic effects of ACE inhibitors: review of current clinical evidence and ongoing clinical trials. Eur Heart) 1998;19 (Suppl J):136-44.

4 Braunwald E, Domanski MJ, Fowler SE, et al. PEACE Trial Investigators. Angiotensin-converting-enzyme inhibition in stable coronary artery disease. N Engl / Med 2004;351:2058-68. doi:10.1056/NEJMoa042739.

5 Pitt B, O’Neill B, Feldman R, et al. QUIET Study Group. The QUinapril Ischemic Event Trial (QUIET): evaluation of chronic ACE inhibitor therapy in patients with ischemic heart disease and preserved left ventricular function. Am J Cardiol 2001;87:1058-63. doi:10.1016 S0002-9149(01)01461-8.

6 Nissen SE, Tuzcu EM, Libby P, et al. CAMELOT Investigators. Effect of antihypertensive agents on cardiovascular events in patients with coronary disease and normal blood pressure: the CAMELOT study: a randomized controlled trial. JAMA 2004;292:2217-25. doi:10.1001/ jama.292.18.2217.

7 Rouleau JL, Warnica W), Baillot R, et al. IMAGINE (Ischemia Management with Accupril post-bypass Graft via Inhibition of the coNverting Enzyme) Investigators. Effects of angiotensin-converting enzyme inhibition in low-risk patients early after coronary artery bypass surgery. Circulation 2008;117:24-31. doi:10.1161/CIRCULATIONAHA.106.685073.

8 Fihn SD, Gardin JM, Abrams J, et al. American College of Cardiology Foundation American Heart Association Task Force on Practice Guidelines American College of Physicians American Association for Thoracic Surgery Preventive Cardiovascular Nurses Association Society for Cardiovascular Angiography and Interventions Society of Thoracic Surgeons. 2012 ACCF/AHA/ACP/AATS/PCNA/SCAI/STS Guideline for the diagnosis and management of patients with stable ischemic heart disease: a report of the American College of Cardiology Foundation/American Heart Association Task Force on Practice Guidelines, and the American College of Physicians, American Association for Thoracic Surgery, Preventive Cardiovascular Nurses Association, Society for Cardiovascular Angiography and Interventions, and Society of Thoracic Surgeons. J Am Coll Cardiol 2012:60:e44-164 doi:10.1016/ijacc. 2012.07.013.

9 Higgins J, Green S. Cochrane Handbook for Systematic Reviews of Interventions Version 5.0.0 ed: Cochrane Collaboration, 2008. www. cochrane-handbook.org.

10 Moher D, Cook DJ, Eastwood S, Olkin I, Rennie D, Stroup DF. Improving the quality of reports of meta-analyses of randomised controlled trials: the QUOROM statement. Quality of Reporting of Meta-analyses. Lancet 1999-354:1896-900 doi:101016/S0140-6736(99)04149-5.

11 DerSimonian R, Laird N. Meta-analysis in clinical trials. Control Clin Trials 1986;7:177-88. doi:10.1016/0197-2456(86)90046-2.

12 Sweeting MJ, Sutton AJ, Lambert PC. What to add to nothing? Use and avoidance of continuity corrections in meta-analysis of sparse data. StatMed 2004;23:1351-75. doi:10.1002/sim.1761

13 Galbraith RF. A note on graphical presentation of estimated odds ratios from several clinical trials. Stat Med 1988;7:889-94. doi:10.1002/sim.4780070807

14 Dias S, Sutton AJ, Welton NJ, Ades AE. Evidence synthesis for decision making 3: heterogeneity-subgroups, meta-regression, bias, and bias-adjustment. Med Decis Making 2013;33:618-40. doi:10.1177/0272989X13485157.

15 Arends LR, Hoes AW, Lubsen J, Grobbee DE, Stijnen T. Baseline risk as predictor of treatment benefit: three clinical meta-re-analyses. Stat Med 2000;19:3497-518. doi:10.1002/1097-0258(20001230)19:24<3497: :AID-SIM830>3.0.CO;2-H

16 Higgins IP, Thompson SG. Controlling the risk of spurious findings from meta-regression. Stat Med 2004;23:1663-82. doi:10.1002/sim.1752.

17 Bradburn MJ, Deeks JJ, Altman DG. Sbe24: metan -an alternative metaanalysis command. Stata Technical Bull Reprints 1998;8:86-100.

18 Thorlund K, Engstrøm J, Wetterslev J, Brok J, Imberger G, Gluud C. User manual for trial sequential analysis (TSA). 2011. www.ctu.dk/tsa.

19 Wetterslev J, Thorlund K, Brok J, Gluud C. Trial sequential analysis may establish when firm evidence is reached in cumulative meta-analysis. J Clin Epidemiol 2008;61:64-75. doi:10.1016/j.jclinepi.2007.03.013.

20 Wetterslev J, Thorlund K, Brok J, Gluud C. Estimating required information size by quantifying diversity in random-effects model meta-analyses. BMC Med Res Methodol 2009;9:86. doi:10.1186/1471-2288-9-86

21 Elliott WJ, Meyer PM. Incident diabetes in clinical trials of antihypertensive drugs: a network meta-analysis. Lancet 2007;369:201-7. doi:10.1016/S0140-6736(07)60108-1.

22 Sorbets E, Labreuche J, Simon T, et al. Renin-angiotensin system antagonists and clinical outcomes in stable coronary artery disease without heart failure. Eur Heart / 2014;35:1760-8. doi:10.1093/ eurheartj/ehu078.

Appendix: Supplementary material 